UCRL-ID-125830

\title{
Immunotherapy of Metastatic Melanoma by Reversal of Immune Suppression
}

M. W. Biggs

J. E. Eiselein

January 21, 1997

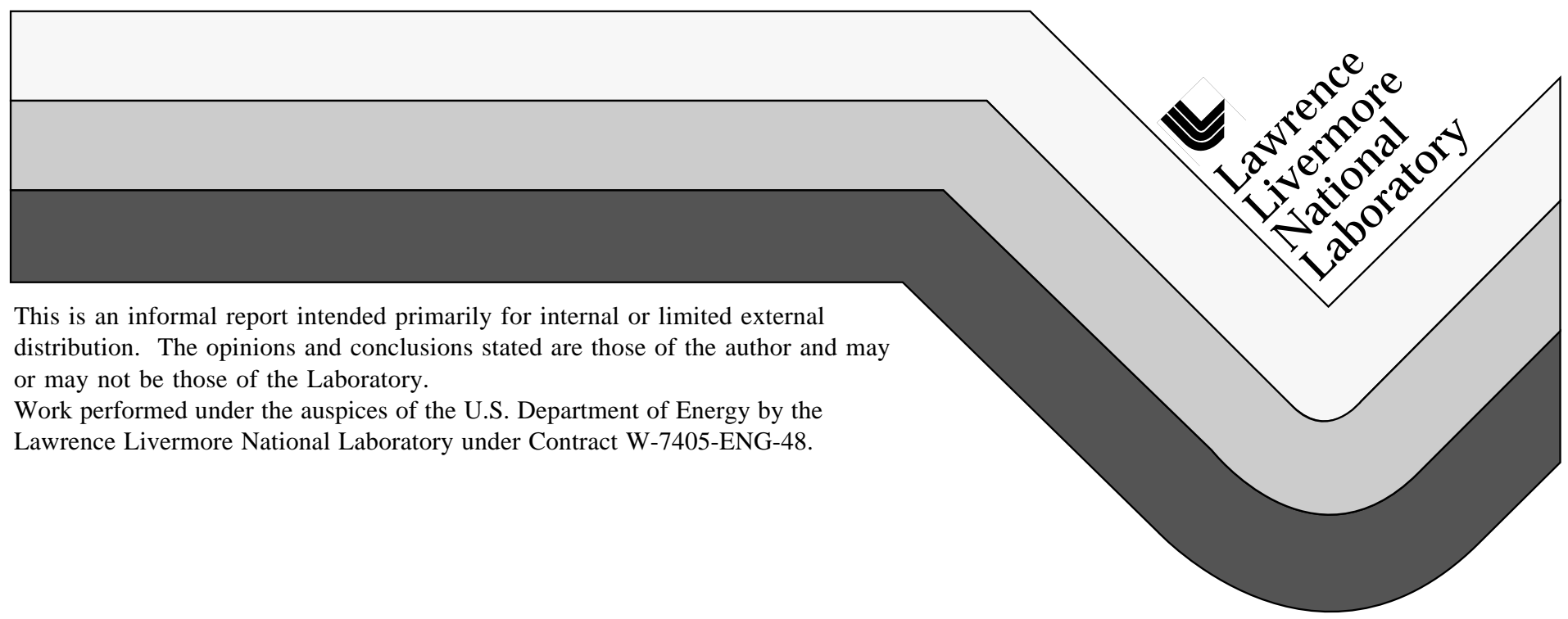




\section{DISCLAIMER}

This document was prepared as an account of work sponsored by an agency of the United States Government. Neither the United States Government nor the University of California nor any of their employees, makes any warranty, express or implied, or assumes any legal liability or responsibility for the accuracy, completeness, or usefulness of any information, apparatus, product, or process disclosed, or represents that its use would not infringe privately owned rights. Reference herein to any specific commercial product, process, or service by trade name, trademark, manufacturer, or otherwise, does not necessarily constitute or imply its endorsement, recommendation, or favoring by the United States Government or the University of California. The views and opinions of authors expressed herein do not necessarily state or reflect those of the United States Government or the University of California, and shall not be used for advertising or product endorsement purposes.

This report has been reproduced directly from the best available copy.

Available to DOE and DOE contractors from the Office of Scientific and Technical Information

P.O. Box 62, Oak Ridge, TN 37831

Prices available from (615) 576-8401, FTS 626-8401

Available to the public from the

National Technical Information Service

U.S. Department of Commerce 5285 Port Royal Rd.,

Springfield, VA 22161 


\title{
IMMUNOTHERAPY OF METASTATIC MELANOMA BY REVERSAL OF IMMUNE SUPPRESSION LDRD 95-ERP-145 January 1997
}

\author{
Max W. Biggs, M.D., Ph.D., and John E. Eiselein
}

The current clinical management of patients with metastatic melanoma is unsatisfactory. Surgery, chemotherapy, and radiation therapy provide little benefit. As a result various immunotherapy protocols are being evaluated in many medical centers world wide. Treatment with various tumor vaccines, adjuvants, adoptive immunotherapy with expanded lymphocytes, and cytokines alone or in combinations has produced encouraging results in some patients. Roughly $20 \%$ of patients treated with various protocols of immunotherapy show various degrees of remission. Some of these remissions are significant, even total. (Ref 1)

The observations reported in this paper are based on the working hypothesis that immune surveillance functions in early melanoma and that metastases are facilitated when this immune surveillance is suppressed. Genetic engineering may prove to be an effective treatment approach for cancer patients ultimately but effective protocols seem to lie in the distant future. We believe that immunotherapy offers the best potential for a useful quality-life extension for advanced melanoma patients in the near future. Effective immunotherapy requires that immune suppression to melanoma antigens be reversed followed by effective antigen presentation in a vaccine and a treatment protocol that prevents reestablishment of suppression.

We view cancer in general and melanoma in particular as a multistage disease requiring some 7 somatic mutations in a single cell. The clinical course of melanoma supports this multistage idea (Ref 2). In addition the observation by Armitage and Doll (Ref 3) that the incidence of cancer in man is a function of the sixth power of age is convincing. The timing sequence of these mutations is probably not restricted but it seems certain that early on mutations that destroy the apoptosis program within the precancerous cell must occur.

\author{
Abbreviations \\ TIL $=$ Tumor Infiltrating Lymphocytes \\ $\mathrm{CTL}=$ Cytotoxic T-cell Response \\ $\mathrm{CY}=$ Cyclophosphamide \\ MTV = Mammary Tumor Virus \\ IL-2 = Interleukin-2 \\ PBL $=$ Peripheral Blood Lymphocytes \\ IVS $=$ In vitro Sensitization \\ MV(50) = Poliovirus Sabin 1 Tumor Cell Lysate filtered through a $50 \mathrm{~nm}$ filter \\ $\mathrm{MV}(25)=$ As above but filtered through a $25 \mathrm{~nm}$ filter to remove the poliovirus \\ Assay $=$ Cr-51, 4 hour, microcytotoxicity assay
}

January 1997

Page 1 of 11 
The essential mechanism whereby cancer is prevented in man undoubtedly involves the intracellular systems that identify significant genetic mutations and activate apoptosis. The second line of defense is the extracellular systems that identify cells with significant genetic mutations and effect cell destruction. NK-LAK and cytotoxic T-cells function within this multifaceted system. These extracellular systems are of particular interest in immunotherapy for they do not depend on intracellular apoptosis systems which are obviously defective in cancer.

In general terms, cancer requires mutations that allow it to:

(1) Escape both intracellular and extracellular cell destruction when the genome becomes abnormal.

(2) Escape extracellular and intracellular "control" signals to grow and not to grow.

(3) Escape the need for adhesion molecules which define an "area code" in order to replicate.

(4) Gain the ability to penetrate capillary epithelium and basement membranes.

We believe one of the essential mutations allows the cancer to escape immune surveillance and establish immune tolerance to tumor antigens. The mode of expression of MHC class 2 molecules on the melanoma cell surface is part of that tolerizing system.

The original proposal that the immune system functions to control cancer is credited to Paul Ehrlich at the beginning of the 20th century. The modern formulation of the hypothesis was presented by Burnett in 1967 (Ref 4). Whether or not the immune system is of significant importance in determining the clinical course of cancer is unsettled and has been debated for many years. Our interpretation of the evidence is that it does operate in "early" cancer and that immune surveillance is suppressed in advanced and metastatic cancer.

The etiology of melanoma is poorly defined. No specific carcinogenic chemicals and no identified viruses have been implicated. The current belief is that melanoma is the result of somatic mutations in the melanotic cells of the skin due to UV exposure in "susceptible" individuals. Such mutated cells due to UV radiation would be predictably antigenic. No melanoma specific antigens have been identified; however, various melanoma associated antigens, primarily glycoproteins and neuroglandular antigens, have been described. (Ref 5) Rosenberg and coworkers (Ref 6) have identified a polypeptide epitope designated MART-1 27-35 which appears to be common to many different melanomas.

The presence of tumor infiltrating lymphocytes (TIL) is frequently found in the histological slides in early melanoma and this has been interpreted as an immune response against melanoma antigens. Indeed the presence of such TILs has been 
shown to be associated with a favorable prognosis (Ref 7). Further, various investigators have shown that peripheral blood lymphocytes from melanoma patients respond in vitro with a cytotoxic T-cell (CTL) response following in vitro stimulation with irradiated autologous melanoma cells. (Ref 8,9)

However, the TILs separated de novo from melanoma tumors do not show an active CTL response. (Ref 10) Why this is so has been investigated extensively and the explanation seems to be that the CD4 T-lymphocyte mix within the tumor milieu is immunosuppressive. Interleukin-10 secretion has been implicated. Usually these TILs which are initially non-cytotoxic, do become cytotoxic when expanded with Interleukin-2 in the presence of irradiated autologous tumor cells. (Ref 11,12)

Immune suppression in lymph nodes draining melanoma sites has been studied and suppression has been found. Of particular interest is the observation that this suppression appears to be localized to the tumor environs and is not systemic. (Ref 13)

Within the T-cell compartments of the immune system there are pathways to initiate and augment both humoral and cytotoxic immune responses. In addition there are T-cell systems that down regulate and control these positive responses. So called "suppressor" lymphocytes have been demonstrated and studied in animals but such cells have not been cloned or identified as to a specific phenotype. These "suppressor" cells are apparently more susceptible to destruction with cyclophosphamide (CY) than are lymphocytes of the cytotoxic branch of the equation. (Ref 14) Numerous experiments have shown that a relatively low dose of CY given before antigen will increase the host's response, both humoral and cytotoxic, over that of control animals. It is thought that suppressor cell function has been destroyed by the CY.

The literature on immune suppression as it relates to melanoma is extensive and an understanding of these data are important to understanding our research efforts. Refer to Chapter 2 "Immune Suppression in Human Melanoma" by Max W. Biggs, John Eiselein and Stanley P.L. Leong in Immunotherapy of Malignant Melanoma, R.G.Landes Co., Austin Texas, 1996. This is a brief 18 page summary of this literature with 60 references.

Some years ago we reported studies on mammary tumors in mice that indicate an approach whereby the suppressor side of the immune equation can be downregulated without destroying the cytotoxic capability. (Ref 15,16) A very brief summary of those studies follows. We isolated a murine enterovirus from a transplanted Ehrlich ascites tumor. This passenger virus apparently had undergone mutational changes which caused it to become cytolytic to the murine mammary tumor cells. The virus caused no apparent disease in our mouse colony (A-Strong) and the colony carried demonstrable humeral antibodies to this virus from birth. This virus readily lysed mammary tumor cells in tissue culture. We prepared a 
virus tumor lysate by serially filtering the tumor lysate down to a $50 \mathrm{~nm}$ Millipore filtration. The preparation was water clear and contained high titers of virus. In homologous ascites tumor transplants intraperitoneal injection of this virus preparation into established ascites tumors lysed the tumor and cured the animals. However, solid subcutaneous transplants of the same tumor cells were not cured with virus alone. We could demonstrate that the virus grew in the solid tumors but regression did not occur. When we gave a single dose of CY 24 hours after the virus injection into the solid tumors they regressed dramatically and the animals were cured. We made similar observations in palpable spontaneous mammary tumors. The A-Strong animals that we used are known to carry MTV and retired breeders develop spontaneous tumors. When we injected the virus tumor lysate into these tumors and followed the injection with CY 24 hours later approximately $50 \%$ of the spontaneous tumors underwent significant regression and some animals were cured.

Our interpretation of these experiments was that the Ehrlich ascites tumor we studied readily established immune suppression to tumor antigens to allow its growth. We believe the virus further participated in this suppressor system. Cell lysis and preparation of a "soluble" antigen, free of particulate tumor antigen, by filtration through the small, 50nm, filter gave an antigen preparation that actively stimulated "suppressor" lymphocytes or the cells secreting suppressor cytokines. Then administration of CY 24 hours later either destroyed those cell, or destroyed the capability of those cells to secrete suppressor cytokines. Important to this idea is that the "soluble" viral tumor lysate did not contain antigen capable of stimulating a CTL response against tumor antigen. We believe we stimulated the "suppressor" Tcell mix but not the CTL capability and then down regulated the suppressor function with CY without destroying the CTL capability. Further we believe that following down regulation of suppression the enteroviral-tumor antigenic complexes functioned anamnistically in these animals to effect the tumor regression. Details of this immune response are not clear but tumor regression occurred before immune tolerance was reestablished. These observations suggest an approach to reversing immune suppression in human cancer.

\section{OBSERVATIONS WITH POLIOVIRUS SABIN 1 IN HUMAN MELANOMA}

It was discovered that the human enterovirus, Poliovirus Sabin 1, will lyse human melanoma cells in tissue culture. This virus cell destruction will kill established melanoma tissue cultures, as well as newly cultured melanoma cells isolated enzymatically from melanoma surgical specimens. Cell death occurs in approximately 24 hours. Expansion of the virus titers and virus replication within the melanoma cells can be demonstrated.

We next turned our attention to demonstrating whether or not a poliovirus melanoma lysate filtered through a $50 \mathrm{~nm}$ filter would suppress an in vitro cytotoxic response against autologous melanoma in a human system. 


\section{DEMONSTRATION OF CYTOTOXICITY AGAINST AUTOLOGOUS MELANOMA}

A standard protocol for demonstrating a CTL response in a patient's peripheral blood lymphocytes against his autologous irradiated melanoma cells was developed.

Peripheral blood lymphocytes were separated from whole blood on a Ficoll-Hypaque gradient. These lymphocytes were cultured in NCTC culture medium containing $100 \mathrm{U} / \mathrm{ml}$ of IL-2 and $10 \%$ fetal bovine serum. In vitro sensitization was done with irradiated, $4500 \mathrm{rad}$, autologous melanoma cells, lymphocyte $/$ melanoma cell ratio $=$ 100/1. NK-LAK and CTL activity were measured using a Cr51, 4 hour, microcytotoxicity assay.

The Cr51 assay was done at 14 days to allow some decay of NK-LAK activity thereby facilitating demonstration of a CTL response. The standard protocol is outlined below.

$\begin{array}{crl}\text { (1) } & \text { DAY 0 } & \text { PBL + IL-2 + IVS } \\ & 7 & \text { IVS + IL-2 } \\ & 14 & \text { ASSAY } \\ \text { (2) } \quad \text { DAY 0 } & \text { PBL + IL-2 } \\ & 1 & \text { IVS } \\ & 7 & \text { IVS + IL-2 } \\ & 14 & \text { ASSAY } \\ & & \\ \text { (3) } \quad \text { DAY 0 } & \text { PBL + IL-2 + MV(50) } \\ & 1 & \text { IVS } \\ & 7 & \text { IVS + IL-2 + MV(50) } \\ & 14 & \text { ASSAY } \\ & & \\ \text { (4) } \quad \text { DAY 0 } & \text { PBL + IL-2 } \\ & 7 & \text { IL-2 } \\ & 14 & \text { ASSAY } \\ & & \\ \text { (5) } & \text { DAY 0 } & \text { PBL + IL-2 + MV(50) } \\ & 7 & \text { IL-2 } \\ & 14 & \text { ASSAY }\end{array}$

Culture \#1 measured the combined cytotoxicity of the CTL response plus the NKLAK response. Culture \#2 measured the combined cytotoxicity of CTL response plus NK-LAK response when IVS was delayed 24 hours. Culture \#3 measured the CTL + NK-LAK cytotoxicity when the lymphocytes were exposed to MV(50) for 24 hours before IVS. Culture \#4 gave a measure of the NK-LAK activity. Culture \#5 measured NK-LAK suppression by MV(50). Assay results from Culture \#2 minus 
results in Culture \#4 gives evidence of a CTL response if present. Culture \#3 gives evidence of suppression of the CTL response by MV(50).

PBLs from 6 patients were examined with respect to their ability to generate a CTL response against their autologous melanoma.

\begin{tabular}{|c|ccc|c|c|c|c|c|}
\hline \multicolumn{2}{|c|}{ Table 1 } \\
\hline $\begin{array}{c}\text { Patient } \\
\text { Culture }\end{array}$ & & Se & Ch & Tu & P r & Ha & M a \\
\hline 1 & $91 \%$ & $64 \%$ & $49 \%$ & & $91 \%$ & $27 \%$ & $75 \%$ & $32 \%$ \\
2 & & $63 \%$ & & $60 \%$ & $82 \%$ & $14 \%$ & $74 \%$ & $37 \%$ \\
3 & $31 \%$ & & & $65 \%$ & $62 \%$ & $13 \%$ & $68 \%$ & $26 \%$ \\
4 & & & $22 \%$ & $104 \%$ & $52 \%$ & $36 \%$ & $62 \%$ & $36 \%$ \\
5 & $34 \%$ & & & $80 \%$ & $50 \%$ & $31 \%$ & $67 \%$ & $31 \%$ \\
\hline
\end{tabular}

Interpretation of the data was as follows. Patient Se was capable of generating a CTL response and $\mathrm{MV}(50)$ prepared from her autologous melanoma was capable of suppressing this CTL response. Patient Ch showed NK-LAK activity but no evidence of a CTL response. Patient Tu showed a CTL response and this CTL response was suppressed by MV(50) prepared from his tumor. Patient Pr showed very little NK-LAK activity and no evidence of a CTL response. Patient Ha had NKLAK activity but no CTL response. Patient Ma showed no evidence of a CTL response.

At this point in our research we felt it expedient to pursue the experiment in a Phase 1 clinical trial in man. We had a "safe" attenuated human enterovirus that would lyse melanoma cells and MV(50) prepared from autologous melanoma cultures appeared to suppress the generation of the CTL response consistent with our basic hypothesis. Application to the Committee for Human Research (CHR) at UCSF, San Francisco was submitted. In that application the protocol included parenteral injection of viable Poliovirus Sabin 1 into cutaneous melanoma metastases followed in 24 hours by the oral administration of cyclophosphamide $(300 \mathrm{mg} / \mathrm{m} 2)$. The patient was to receive a booster dose of "Salk type" killed poliovaccine, IPOL, prior to the experiment and we would demonstrate neutralizing antibody in the patient's serum prior to proceeding. It was pointed out that the patients had very limited life expectancy. The CHR rejected the application, fearing that the parenteral injection of viable Poliovirus Sabin 1 virus might cause poliomyelitis even though the patient had been immunized against the virus and the virus was an attenuated strain used for oral immunization in infants.

Rather than abandon the experiment entirely we decided to remove the virus by filtration through a $25 \mathrm{~nm}$ filter $(\mathrm{MV}(25))$. It was reasoned that the $\mathrm{MV}(25)$ might still contain "soluble" antigen capable of stimulating the "suppressor" cell function. We demonstrated that the virus could be removed completely by this filtration step 
and the MV(25) did appear to be suppressive in our in vitro test system. A second application to the CHR was approved allowing us to inject the MV(25) into cutaneous melanoma metastases followed by the administration of a low dose of CY.

The first patient (ML) treated was a 62 year old Chinese female whose initial surgery was 14 months before referral to the Mt. Zion Melanoma Clinic. Initially she presented to her doctor with a pink macule on the vortex of her scalp. The lesion was excised and the path report was malignant melanoma, nodular type, $6 \mathrm{~mm}$ invasion, Clark level 5, low mitotic rate, absent tumor infiltrating lymphocytes, high risk primary. A second surgery with wide margins was done.

Five months after initial surgery cervical nodes were positive for metastatic melanoma. At 7 months 2 recurrent satellite nodules were removed from the scalp. Cat scans and x-rays were negative for pulmonary metastases. The patient elected not to submit to $\mathrm{x}$-ray therapy or chemotherapy.

When first seen at Mt. Zion, 14 months after initial surgery, the patient had 7 palpable nodules at the primary site and down the right side of the neck to the clavicular area. Cat scans and x-rays showed 4 pulmonary metastases 0.4 to $1.0 \mathrm{~cm}$ in diameter.

The patient gave informed consent and was entered into the protocol. A cervical tumor nodule was excised, and approximately 40x10(6) viable tumor cells were isolated enzymatically. These cells were placed in tissue culture. At 24 hours all unattached cells were removed. At 2 days the cultures were infected with poliovirus Sabin 1 and the cells were placed in culture medium free of fetal bovine serum. At 4 days the cells showed extensive cytopathological changes. The cells and culture medium was freeze thawed 3 times and filtered serially down to $25 \mathrm{~nm}$ porosity. This $\mathrm{MV}(25)$ was assayed for the presence of infectious virus. It was necessary to filter the lysate a second time through the $25 \mathrm{~nm}$ filter before all infectious virus was removed. The patient was vaccinated with IPOL parenterally and 7 days later the patient's serum was positive for neutralizing antibodies against Poliovirus Sabin 1. Assay of the patient's peripheral blood lymphocytes for the ability to generate a cytotoxic T-cell response after in vitro sensitization with autologous melanoma was negative.

At treatment day 0 the 7 palpable nodules were measured, width and breadth, and injected with $\mathrm{MV}(25), 1.5 \mathrm{ml}$ total, containing the lysate from approximately $3 \times 10(6)$ melanoma cells. At day +1 she received $480 \mathrm{mg}$ of cyclophosphamide p.o. (300 $\mathrm{mg} / \mathrm{m}(2))$. She experienced no adverse symptoms from the treatment. Initially the mean size of the injected lesions was $73 \pm 23 \mathrm{~mm}(2)$, day $+5=55 \pm 13 \mathrm{~mm}(2)$, day +12 $=58 \pm 19 \mathrm{~mm}(2)$, and day $+20=66 \pm 22 \mathrm{~mm}(2)$. At day +12 a new nodule $4 \mathrm{~mm}(2)$ was noted and this increases on day +20 to $20 \mathrm{~mm}(2)$. In view of the apparent absence of significant response the patient was entered into a second separate immunotherapy protocol at day 21. 
A second patient was treated. This was a 58 year old man who had had initial surgery for a melanoma on his back 14 months prior to being seen at Mt Zion. The primary was a nodular melanoma, $3.72 \mathrm{~mm}$ thick, Clark level 3-4. Three months prior to being seen he had developed satellite subcutaneous metastases about the primary surgical scar and a Cat scan showed brain metastases.

After informed consent two tumor biopsy specimens were taken, one from the back and one from the axilla. These were combined and approximately 40x10(6) melanoma cells were separated enzymatically and cultured in 11 culture flasks. These cells were lysed with poliovirus Sabin 1, freeze thawed 3x, and filtered through a $25 \mathrm{~nm}$ filter as described above for patient \#1. The $19 \mathrm{ml}$ of $\mathrm{MV}(25)$ obtained was concentrated by filtration over a filter with a cut off at $10 \mathrm{kDa}$ molecular weight to $2.6 \mathrm{ml}$. This preparation was free of infectious virus and was used for treatment.

After the patient had received IPOL poliovaccine and neutralizing antibodies had been demonstrated in his serum, 10 subcutaneous lesions on the back were measured and injected with the MV(25). The mean of the 10 treated lesions was $394 \pm 70 \mathrm{~mm}(2)$. Twenty-four hours later the patient was given CY, $300 \mathrm{mg} / \mathrm{m}(2)$, by mouth. The patient experienced no adverse symptoms from the treatment. Periodic observation following treatment revealed no decrease in the size of any of the treated lesions and no inflammatory change. The patient was treated with gamma-knife radiotherapy for complications of his brain metastases on treatment day +60 and he died on treatment day +75 .

After these two patients the decision was reached to abandon the clinical trial. It was felt the MV(25) without virus and apparently only a trace of antigenic protein had no chance for success in the very advanced melanoma patients we were trying to treat.

\section{CONCLUSION}

We set out to prove or disprove the hypothesis that we could differentially down regulate the suppressor side of the immune system with our protocol to allow a cytotoxic response. We did not succeed. However, it is to be emphasized we were not permitted to repeat the essential features of the murine experiment in man. We believe both live virus and "solubilized" antigen in quantity are essential for success in this approach.

Our hypothesis remains a viable approach to the reversal of suppression to allow a CTL response in cancer patients. 
Five peripheral, preliminary observations made in the course of this research warrant further study. These observations should be expanded when research monies are available.

(1) $M V(50)$ prevents the expected generation of a cytotoxic response in peripheral blood lymphocytes on in vitro stimulation with irradiated autologous melanoma cells. It appears that the lymphocytes must be exposed to the "soluble" antigen for a period of time before exposure to the autologous melanoma antigen. If the MV(50) and IVS with autologous melanoma are initiated together at Day 0 suppression does not occur. The nature of this suppression should be studied and defined as to phenotype of the suppressor cell mix and the cytokines involved. It has been shown that suppression is not due to poliovirus destruction of essential immune cells. The data seems to argue against a simple competition for antigen sites.

(2) Infection of melanoma cells which express MHC-class 2 molecules on their surface is followed by rapid disappearance of those surface molecules. It is known that many melanomas abnormally express MHC-class 2 molecules on their surface. When such cells are infected in tissue culture with poliovirus, there is a complete loss of these surface molecules within 4 hours. It is likely that the molecules are released and "solubilized". Release of such molecules in vivo would result in immune tolerance.

This observation suggests the hypothesis that were a "controlled" non-growing melanoma to be infected in vivo with an enterovirus, the release of surface antigens might establish tolerance which would then allow renewed tumor growth.

(3) In one patient the ability to generate an in vitro cytotoxic response in peripheral blood lymphocytes as a result of in vitro autologous tumor sensitization was measured when first seen and then 6 months later after her disease had progressed. She was able to generate a cytotoxic response initially but this capacity disappeared 6 months later. The target melanoma was identical in the 2 measurements for the target cells in both assays were from the biopsy done when she was first seen and preserved in the frozen state. It has been proposed that changes in the MHC class 1 antigenic presentation of metastatic melanoma with time is the best explanation why patients are unable to generate a cytotoxic response as the disease progresses. Our observation indicates that in this one case the inability to respond immunologically as the disease progressed was due to tolerization for the target melanoma cells were identical in the two assays.

(4) MV(50) from Patient A will suppress the CTL response in Patient B if they share identical HLA class 2 molecules. PBLs from a patient capable of generating a CTL response were exposed to $\mathrm{MV}(50)$ from 3 patients who were not capable of a CTL response. Two of the MV(50)s did not suppress while one did. The two that did not suppress did not share any HLA class 2 molecules with the patient capable of a CTL response while the patient that suppressed did share DQ7. This preliminary 
observation should be expanded. It argues that the polypeptide antigen in the MHC class 2 molecules in the 2 patients was identical.

(5) It has been reported that patients who show areas of regression in their pathological slides have a poor prognosis. The likely explanation for this baffling observation lies within our hypothesis. These patients likely express MHC class 2 molecules on the surface of their melanoma. The cytotoxic response producing the areas of regression releases solubilized MHC class 2 molecules in quantity. This results in systemic tolerization to the melanoma antigens and the CTL response ceases and tumor growth returns. All such patients are systemically tolerized to melanoma antigens and when compared to melanoma patients as a whole many of which are not systemically tolerized they have a poorer prognosis.

\section{REFERENCES}

(1) Leong SPL, ed. Immunotherapy of Malignant Melanoma. Austin: RG Landes 1996.

(2) Sagebiel R., Epidemiology and pathology of malignant melanoma. In: Leong SPL, ed. Malignant melanoma: advances in treatment. Austin: RG Landes 1992:1-17.

(3) Armitage A, Doll R. The age distribution of cancer and a multi-stage theory of carcinogenesis. British J Cancer 1954; 8:1-12.

(4) Burnet M. Immunologic aspects of malignant disease. Lancet 1967; 1:1171.

(5) Graf Jr LH, Ferrone S. Human melanoma-associated antigens. Immunol Ser 1989; 43:643-79.

(6) Rivoltini l, Kawakami Y, Sakaguchi k, et al. Induction of tumor-reactive CTL from peripheral blood and tumor infiltrating lymphocytes of melanoma patients by in vitro stimulation with an immunodominant peptide of the human melanoma antigen MART-1. J Immunol 1995; 154:2257-2265.

(7) Clark Jr WH, Elder DE, Guerry IV D et al. Model predicting survival in stage 1 melanoma based on tumor progression. J Nat Cancer Inst 1989; 81:1893-1904.

(8) Leong SPL, Zhou YM, Granberry ME, et al. Generation of cytotoxic effector cells against human melanoma. Cancer Immunology and Immunotherapy 1995; 40:397409 .

(9) Mukherji B, MacAlister TJ. Clonal analysis of cytotoxic T cell response against human melanoma. J Exp Med 1983; 58:240-245 
(10) Holmes EC. Immunology of tumor infiltrating lymphocytes. Ann Surg 1985; 201:158-163.

(11) Tapalian SL, Solomon D, Rosenberg SA. Tumor specific cytolysis by lymphocytes infiltrating human melanoma. J Immunol 1989; 142:3714-3725.

(12) Mathiot C, Robin E, Gey A, et al. Phenotypic and functional analysis of tumourinfiltrating lymphocytes from patients with melanoma and other metastatic cancers. Eur J Cancer 1992; 28:345-350.

(13) Cochran AJ, Pihl E, Wen DR, et al. Zoned immune suppression of lymph nodes draining malignant melanoma: histologic and immunohistologic studies. JNCI 1987; 78:399-405.

(14) Ozer H, Cowens JW, Colvin M, et al. In vitro effects of 4hydroperoxycyclophosphamide on human immunoregulatory $\mathrm{T}$ subset function. J Exp Med 1982; 155:276-290.

(15) Biggs MW, Eiselein JE. Observations on the treatment of autochthonous tumors in mice with an enterovirus and cyclophosphamide. Cancer Letters 1993; 69:133-137.

(16) Eiselein JE, Biggs MW. Treatment of transplanted murine tumors with an oncolytic virus and cyclophosphamide. Cancer Research 1978; 38:3817-3822. 


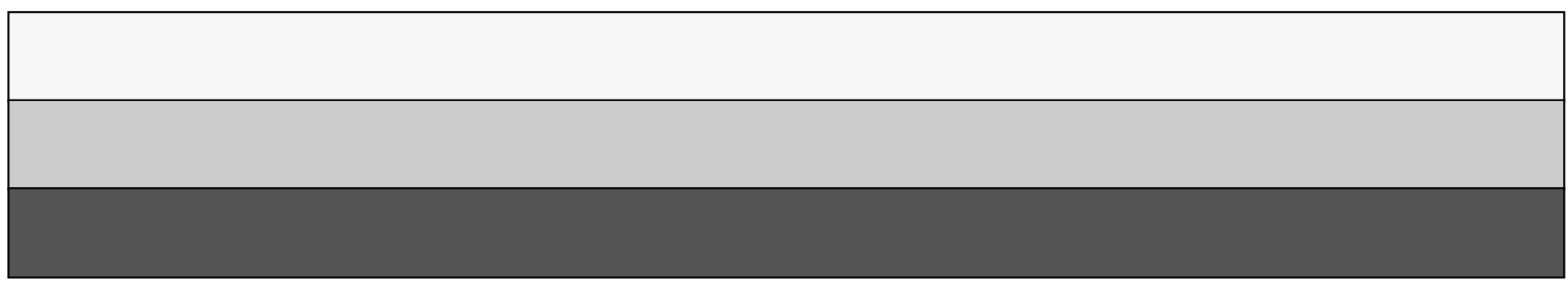

